\title{
DIMENSIONAMENTO, SIMULAÇÃO E ANÁLISE DE SENSIBILIDADE DE SISTEMAS DE EVAPORAÇÃO MÚLTIPLO EFEITO DE INDÚSTRIAS DE CELULOSE KRAFT
}

\author{
J. M. GASPARONI ${ }^{1,1}$, L. A. F. OLIVEIRA ${ }^{1}$, M. O. AGUIAR ${ }^{2}$, \\ C. R. S. N. ALMEIDA ${ }^{3}$ e G. M. ALMEIDA ${ }^{2}$ \\ ${ }^{1}$ Universidade Federal de São João del-Rei, Departamento de Engenharia Química e \\ Estatística \\ ${ }^{2}$ Universidade Federal de Minas Gerais, Departamento de Engenharia Química \\ ${ }^{3}$ Universidade Federal de São João del-Rei, Departamento das Engenharias de \\ Telecomunicações e de Mecatrônica; e Centro Federal de Educação Tecnológica de Minas \\ Gerais, Departamento de Engenharia Elétrica \\ E-mail para contato: galmeida@deq.ufmg.br
}

\begin{abstract}
RESUMO - Com regulações cada vez mais restritivas da sociedade, dos governos e de associações, é crescente o papel da Engenharia de Processos para a melhoria dos processos, o atendimento às legislações e a manutenção e ganho de mercado. No presente trabalho, após a modelagem matemática e validação de um sistema de evaporação múltiplo efeito de indústrias de celulose Kraft, exploraram-se as suas etapas de dimensionamento de equipamentos, de simulação de novos cenários e de análise de sensibilidade de variáveis de interesse.
\end{abstract}

\section{INTRODUÇÃO}

A Engenharia de Processos constitui-se em um procedimento sistematizado para o projeto de processos químicos industriais. As suas etapas: dimensionamento de equipamentos, simulação de cenários operacionais e análise de sensibilidade sobre variáveis de interesse, respondem, de modo satisfatório, a diversas questões sobre os processos (Perlingeiro, 2005). Com a maior complexidade dos processos industriais, e as regulações mais restritivas da sociedade, governos e associações, a demanda por esse conjunto de análises é crescente. Atualmente, um maior entendimento das operações é uma questão essencial para a própria manutenção do negócio. Neste trabalho, exploraram-se cada uma dessas etapas, utilizando-se um sistema de evaporação múltiplo efeito de indústrias de celulose Kraft como estudo de caso (Figura 1). Este sistema é responsável por concentrar o licor negro, subproduto da etapa de polpação dos cavacos de madeira, inicialmente entre 15 e $20 \%$ em massa, acima de $60 \%$ (neste trabalho, adotou-se 50\%). O número usual de efeitos é seis, sendo o primeiro efeito composto por três ou quatro evaporadores (neste trabalho, adotou-se um evaporador por efeito). A alimentação do licor diluído ocorre entre o quarto e o sexto efeitos (neste trabalho, adotou-se o quinto efeito), e a saída do licor concentrado e a entrada do vapor vivo (fonte externa) são no primeiro efeito (SENAI e IPT, 1988; Venkatesh e Nguyen, 1985).

\footnotetext{
${ }^{1}$ In memoriam.
} 
Figura 1 - Sistema de evaporação de múltiplo efeito.

(Fonte: Kumar et al. (2013))

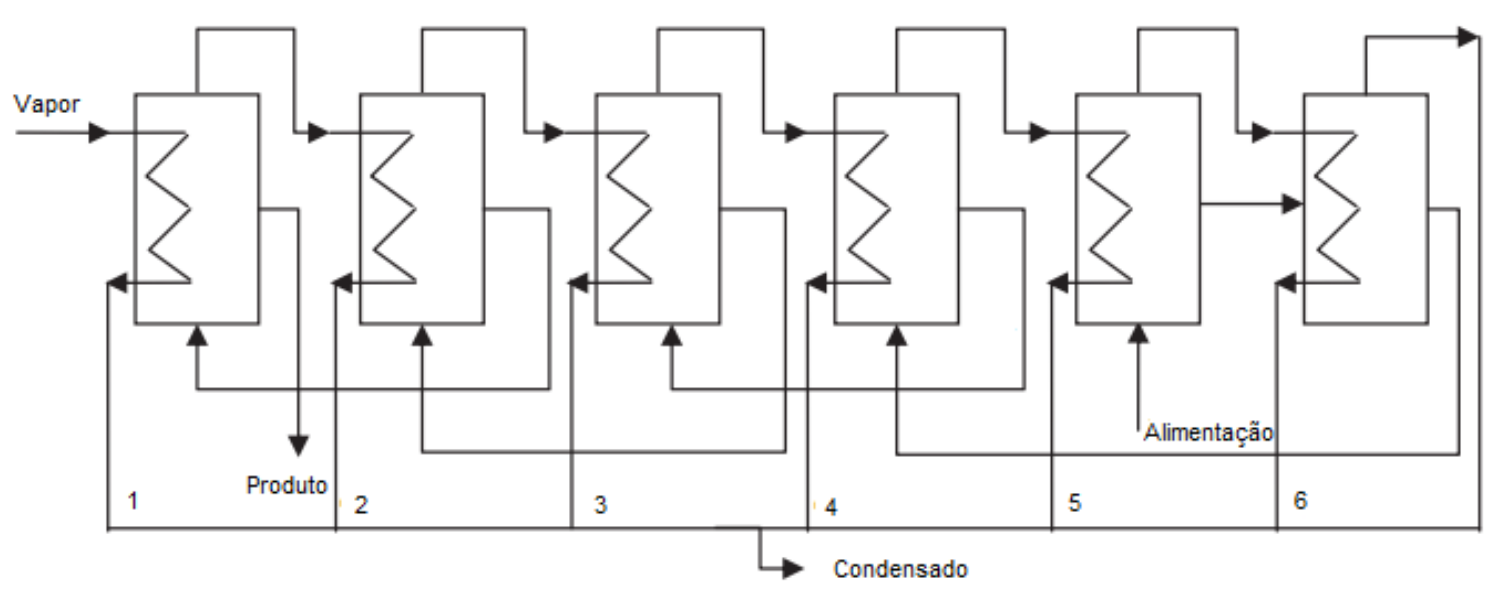

\section{OBJETIVOS}

Aplicar os conceitos de engenharia de processos, com os objetivos de dimensionamento, de simulação e de análise de sensibilidade. Utilizou-se o sistema de evaporação múltiplo efeito de indústrias de celulose Kraft como estudo de caso. Empregou-se o software $\mathrm{MATLAB}^{2}$ para a automatização dos cálculos.

\section{METODOLOGIA}

Descrevem-se, a seguir, as etapas da metodologia. (Etapas preparatórias) Após o reconhecimento do processo (Figura 1), realiza-se a sua modelagem matemática. Utilizaramse as equações de balanço de massa para o vapor (Equação 1), o licor (Equação 2) e o soluto do licor (Equação 3); as equações de balanço de energia para o vapor (Equação 4) e o licor (Equação 5); e a equação global de transferência de calor (Equação 6), em que $\dot{m}=$ vazão mássica $(\mathrm{kg} / \mathrm{s}), x=$ concentração, $H=$ entalpia específica $(\mathrm{kJ} / \mathrm{kg}), q=$ taxa de transferência de calor $(\mathrm{kJ} / \mathrm{s}), U=$ coeficiente global de transferência de calor $\left(\mathrm{W} /\left(\mathrm{m}^{2} \cdot{ }^{\circ} \mathrm{C}\right)\right), A=$ área de troca térmica $\left(\mathrm{m}^{2}\right), T=$ temperatura $\left({ }^{\circ} \mathrm{C}\right)$, e os subscritos $e, s$ e sat, entrada, saída e saturação, respectivamente, conforme McCabe et al. (2005). Ainda calcularam-se os índices usuais de desempenho: economia de vapor (= $m a s s a_{\text {água evaporada }} / m_{a s s} a_{\text {vapor vivo }}$ ), capacidade de evaporação (=masság á evaporada $/ \Delta t$ ) e consumo de vapor (=capacidade/economia), conforme McCabe et al. (2005). Considerações (conforme Kumar et al. (2013)): (1) Vapor de saída como líquido saturado (a partir das Equações 1 e $4, q_{s}=\dot{m} \cdot \lambda$, em que $\dot{m}_{e}=\dot{m}_{s}=\dot{m}$ e $\lambda=$ calor latente); (2) Perda de calor desprezível $\left(q_{s}=q_{e}\right)$; (3) Não há perda de sólidos do licor (Equação 3); (4) Coeficiente global de transferência de calor constante ([1160 (efeito 1), $1220,1280,1335,1365,1400$ (efeito 6$)] \mathrm{W} /\left(\mathrm{m}^{2} \cdot{ }^{\circ} \mathrm{C}\right)$ ); (5) Configuração do fluxo do licor: 5 (entrada do licor diluído) $\rightarrow 6 \rightarrow 4 \rightarrow 3 \rightarrow 2 \rightarrow 1$ (entrada do vapor vivo e saída do licor concentrado), conforme a Figura 1. A etapa seguinte refere-se a estimativa de propriedades $e$ coeficientes. Para as propriedades do licor, a saber, massa específica $\left(\mathrm{kg} / \mathrm{m}^{3}\right)$, calor específico $(\mathrm{kJ} /(\mathrm{kg} \cdot \mathrm{K}))$, viscosidade dinâmica $(\mathrm{Pa} \cdot \mathrm{s})$, entalpia específica $(\mathrm{kJ} / \mathrm{kg})$, e elevação do ponto de

\footnotetext{
2 Aquisição de licença pelo Projeto TEC APQ-02544-09 (Edital 01/2009 Universal FAPEMIG).
} 
ebulição (EPE), empregaram-se as correlações experimentais em Holmlund e Parviainen (1999) e Masse et al. (1986). As propriedades do licor são função de sua concentração e temperatura. Para o cálculo das propriedades da água, a saber, entalpia da água evaporada $(\mathrm{kJ} / \mathrm{kg})$, entalpia do vapor saturado $(\mathrm{kJ} / \mathrm{kg})$, e calor latente de vaporização $(\mathrm{kJ} / \mathrm{kg})$, utilizaramse as relações também em Holmlund e Parviainen (1999). (Etapas executivas; seção 4) Passou-se então ao dimensionamento, ou seja, ao cálculo das áreas dos evaporadores, e a simulação, com a simulação de novos cenários de operação, a partir de perturbações na vazão de vapor vivo. $\mathrm{Na}$ sequência, realizou-se um estudo de análise de sensibilidade sobre a concentração final do licor.

$$
\begin{aligned}
& \dot{m}_{e}=\dot{m}_{s} \\
& \dot{m}_{e}=\dot{m}_{s}+\left(\dot{m}_{e}-\dot{m}_{s}\right) \\
& \dot{m}_{e} \cdot x_{e}=\dot{m}_{s} \cdot x_{s} \\
& \dot{m}_{e} \cdot H_{e}=\dot{m}_{s} \cdot H_{s}+q_{s} \\
& \dot{m}_{e} \cdot H_{e}+q_{e}=\dot{m}_{s} \cdot H_{s}+\left(\dot{m}_{e}-\dot{m}_{s}\right) \cdot H_{s} \\
& q=U \cdot A \cdot\left(T_{\text {vapor }, \text { sat }}-T_{\text {licor }, s}\right)
\end{aligned}
$$

\section{APRESENTAÇÃO E DISCUSSÃO DE RESULTADOS}

A etapa de modelagem matemática resultou em 13 equações (1 equação de balanço de massa e 1 equação de balanço de energia para cada um dos 6 efeitos, além da equação global de transferência de calor) e 19 variáveis, conforme procedimento em McCabe et al. (2005). Com a especificação de 6 variáveis (Tabela 1), o número de incógnitas é 13, e o número resultante de graus de liberdade, zero. Portanto, tanto para o dimensionamento quanto para a

\begin{tabular}{|c|c|c|c|}
\hline Dimensionamento & Valor & Simulação & Valor \\
\hline Fluxo de licor(e) $(\mathrm{kg} / \mathrm{s})^{(1)}$ & 23,98 & Fluxo de licor(e) $(\mathrm{kg} / \mathrm{s})^{(1)}$ & 23,98 \\
\hline Concentração do licor(e) $(\mathrm{kg} / \mathrm{kg})^{(1)}$ & 0,1 & Concentração do licor(e) $(\mathrm{kg} / \mathrm{kg})^{(1)}$ & 0,1 \\
\hline Temperatura do licor(e) $\left({ }^{\circ} \mathrm{C}\right)^{(1)}$ & 80 & Temperatura do licor $(\mathrm{e})\left({ }^{\mathrm{o}} \mathrm{C}\right)^{(1)}$ & 80 \\
\hline Pressão do vapor vivo $(\mathrm{Pa})^{(1)}$ & $3,510 \cdot 10^{5}$ & Pressão do vapor vivo $(\mathrm{Pa})^{(1)}$ & $3,510 \cdot 10^{5}$ \\
\hline Concentração do licor(s) (kg/kg) & 0,5 & $\begin{array}{l}\text { Fluxo de vapor vivo }(\mathrm{kg} / \mathrm{s}) \\
(\text { perturbação de }+5 \%)^{(1)}\end{array}$ & 4,4586 \\
\hline Pressão de operação ( sexto efeito) $(\mathrm{Pa})^{(2)}$ & 11727 & $\begin{array}{l}\text { Área de troca térmica } \\
\text { (de cada evaporador) }\left(\mathrm{m}^{2}\right)^{(1)}\end{array}$ & 554,4000 \\
\hline
\end{tabular}
simulação, tem-se um problema determinado sem otimização, cada um deles com um sistema particular de equações não-lineares. Utilizou-se a função $f$ solve do MATLAB para a resolução de ambos.

Tabela 1 - Variáveis especificadas: Dimensionamento (Kumar et al., 2013) e Simulação (Kumar et al. (2013) e seção 4.1), em que (e) = entrada e (s) = saída.

\footnotetext{
${ }^{(1)}$ Condições conhecidas.

${ }^{(2)}$ Metas de projeto e de operação.
} 


\section{Congresso Brasileiro de Engenharia Química \\ em Iniciação Científica \\ UFSCar - São Carlos - SP \\ 16 a 19 de Julho de 2017}

\subsection{Dimensionamento}

A Tabela 2 contém o resultado da etapa de dimensionamento. Validou-se esse resultado, e, portanto, o modelo matemático (seção 3), ao compará-lo com aquele em Kumar et al. (2013). (Adotou-se nas Tabelas 1, 2 e 3 o mesmo número de algarismos significativos em Kumar et al. (2013); ao longo do presente texto, usam-se valores aproximados.) Observa-se uma área de troca térmica de cada evaporador de $554,4 \mathrm{~m}^{2}$, e uma vazão mássica de vapor vivo, dada a especificação de $50 \%$ para o licor concentrado, de 4,2 kg/s.

Tabela 2 - Resultado e validação do dimensionamento (em negrito: variáveis especificadas

(Tabela 1); entre parêntesis: resultado de Kumar et al. (2013); (e) = entrada e (s) = saída)

\begin{tabular}{|c|c|c|c|c|c|c|}
\hline Variável & $\mathbf{I}$ & II & III & IV & $\mathbf{V}$ & VI \\
\hline \multicolumn{7}{|l|}{ Licor } \\
\hline Concentração(e) $(\mathrm{kg} / \mathrm{kg})$ & $\begin{array}{c}0,2806 \\
(0,2883) \\
\end{array}$ & $\begin{array}{c}0,2013 \\
(0,2067) \\
\end{array}$ & $\begin{array}{c}0,1606 \\
(0,1639) \\
\end{array}$ & $\begin{array}{c}0,1384 \\
(0,1401) \\
\end{array}$ & 0,1 & $\begin{array}{c}0,1151 \\
(0,1157) \\
\end{array}$ \\
\hline Concentração(s) (kg/kg) & 0,5 & $\begin{array}{c}0,2806 \\
(0,2883) \\
\end{array}$ & $\begin{array}{c}0,2013 \\
(0,2067) \\
\end{array}$ & $\begin{array}{c}0,1606 \\
(0,1639) \\
\end{array}$ & $\begin{array}{c}0,1151 \\
(0,1157) \\
\end{array}$ & $\begin{array}{c}0,1384 \\
(0,1401) \\
\end{array}$ \\
\hline Vazão(e) (kg/s) & $\begin{array}{c}8,5457 \\
(8,3173)\end{array}$ & $\begin{array}{c}11,9117 \\
(11,6031)\end{array}$ & $\begin{array}{l}14,9256 \\
(14,627)\end{array}$ & $\begin{array}{c}17,3247 \\
(17,1105)\end{array}$ & 23,98 & $\begin{array}{c}20,8200 \\
(20,7300)\end{array}$ \\
\hline Vazão(s) (kg/s) & $\begin{array}{c}4,7960 \\
(4,7960) \\
\end{array}$ & $\begin{array}{c}8,5457 \\
(8,3173) \\
\end{array}$ & $\begin{array}{c}11,9117 \\
(11,6031) \\
\end{array}$ & $\begin{array}{l}14,9256 \\
(14,627) \\
\end{array}$ & $\begin{array}{c}20,8200 \\
(20,7300) \\
\end{array}$ & $\begin{array}{r}17,3247 \\
(17,1105) \\
\end{array}$ \\
\hline Temperatura $(\mathrm{e})\left({ }^{\circ} \mathrm{C}\right)$ & $\begin{array}{c}98,2855 \\
(99,4662)\end{array}$ & $\begin{array}{c}82,5867 \\
(83,4384)\end{array}$ & $\begin{array}{c}70,4266 \\
(71,0077)\end{array}$ & $\begin{array}{l}50,3945 \\
(50,419)\end{array}$ & 80 & $\begin{array}{c}61,1306 \\
(61,3928)\end{array}$ \\
\hline Temperatura $(\mathrm{s})\left({ }^{\circ} \mathrm{C}\right)$ & $\begin{array}{c}124,7940 \\
(125,2157)\end{array}$ & $\begin{array}{c}98,2855 \\
(99,4362)\end{array}$ & $\begin{array}{c}82,5867 \\
(83,4384)\end{array}$ & $\begin{array}{c}70,4266 \\
(71,0077)\end{array}$ & $\begin{array}{c}61,1306 \\
(61,3928)\end{array}$ & $\begin{array}{c}50,3945 \\
(50,419)\end{array}$ \\
\hline $\operatorname{EPE}\left({ }^{\circ} \mathrm{C}\right)$ & $\begin{array}{c}14,1631 \\
(14,1838)\end{array}$ & $\begin{array}{c}4,9192 \\
(5,1611) \\
\end{array}$ & $\begin{array}{c}2,7591 \\
(2,8805)\end{array}$ & $\begin{array}{c}1,8824 \\
(1,9428)\end{array}$ & $\begin{array}{c}1,1405 \\
(1,1489)\end{array}$ & $\begin{array}{c}1,394 \\
(1,419)\end{array}$ \\
\hline Calor específico $(\mathrm{kg} / \mathrm{kg} \cdot \mathrm{K})$ & $\begin{array}{c}3,6404 \\
(3,3006) \\
\end{array}$ & $\begin{array}{c}3,7804 \\
(3,6275) \\
\end{array}$ & $\begin{array}{c}3,8572 \\
(3,7704) \\
\end{array}$ & $\begin{array}{c}3,8962 \\
(3,8507) \\
\end{array}$ & $\begin{array}{c}3,9913 \\
(3,9506) \\
\end{array}$ & $\begin{array}{c}3,9516 \\
(3,8924) \\
\end{array}$ \\
\hline \multicolumn{7}{|l|}{ Vapor } \\
\hline Temperatura $(\mathrm{e})\left({ }^{\circ} \mathrm{C}\right)$ & 139 & $\begin{array}{c}110,6310 \\
(111,0319) \\
\end{array}$ & $\begin{array}{c}93,3663 \\
(94,3051) \\
\end{array}$ & $\begin{array}{c}79,8276 \\
(80,5579) \\
\end{array}$ & $\begin{array}{c}68,5442 \\
(69,0649) \\
\end{array}$ & $\begin{array}{r}59,9900 \\
(60,2439) \\
\end{array}$ \\
\hline Temperatura $(\mathrm{s})\left({ }^{\circ} \mathrm{C}\right)$ & $\begin{array}{c}110,6310 \\
(111,0319)\end{array}$ & $\begin{array}{c}93,3663 \\
(94,3051)\end{array}$ & $\begin{array}{c}79,8276 \\
(80,5579)\end{array}$ & $\begin{array}{c}68,5442 \\
(69,0649)\end{array}$ & $\begin{array}{c}59,9900 \\
(60,2439)\end{array}$ & 49 \\
\hline Vazão(e) (kg/s) & $\begin{array}{c}4,2463 \\
(3,8028) \\
\end{array}$ & $\begin{array}{c}3,7497 \\
(3,5213) \\
\end{array}$ & $\begin{array}{c}3,3659 \\
(3,2858) \\
\end{array}$ & $\begin{array}{c}3,0139 \\
(3,0239)\end{array}$ & $\begin{array}{c}2,3991 \\
(2,4835) \\
\end{array}$ & $\begin{array}{c}3,1500 \\
(3,2500) \\
\end{array}$ \\
\hline Vazão(s) (kg/s) & $\begin{array}{c}3,7497 \\
(3,5213)\end{array}$ & $\begin{array}{c}3,3659 \\
(3,2858)\end{array}$ & $\begin{array}{c}3,0139 \\
(3,0239)\end{array}$ & $\begin{array}{c}2,3991 \\
(2,4825)\end{array}$ & $\begin{array}{c}3,1500 \\
(3,2500)\end{array}$ & $\begin{array}{c}3,5010 \\
(3,6195)\end{array}$ \\
\hline \multicolumn{7}{|l|}{ Índice de desempenho } \\
\hline Área $\left(\mathrm{m}^{2}\right)$ & $\begin{array}{c}554,4000 \\
(591,9614) \\
\end{array}$ & - & - & - & - & - \\
\hline Consumo de vapor $(\mathrm{kg} / \mathrm{s})$ & $\begin{array}{c}4,2463 \\
(3,8028)\end{array}$ & - & - & - & - & - \\
\hline $\begin{array}{c}\text { Capacidade de evaporação } \\
(\mathrm{kg} / \mathrm{s})\end{array}$ & $\begin{array}{l}19,1796 \\
(19,184)\end{array}$ & - & - & - & - & - \\
\hline Economia de vapor $(\mathrm{kg} / \mathrm{kg})$ & $\begin{array}{c}4,5167 \\
(5,0447)\end{array}$ & - & - & - & - & - \\
\hline
\end{tabular}




\subsection{Simulação}

Empregou-se o modelo validado para a simulação de cenários operacionais, a partir de perturbações na vazão de vapor vivo de $\pm 5 \%$. A Tabela 1 mostra as variáveis especificadas, e a Tabela 3 , o resultado para o cenário de $+5 \%$. Observa-se que a concentração final do licor aumenta de 50\% (caso-base) para 62,9\% (cenário 1: $+5 \%$ ) e diminui para $36,2 \%$ (cenário 2 : $-5 \%$; não mostrado por limitação de espaço), dada a área de troca térmica de $554,4 \mathrm{~m}^{2}$. Já a capacidade de evaporação da planta aumenta para $21,1 \mathrm{~kg} / \mathrm{s}$ (cenário 1) e diminui para $17,4 \mathrm{~kg} / \mathrm{s}$ (cenário 2) em relação ao caso-base de 19,2 kg/s. Ainda, a Figura 2(a) mostra o aumento da massa específica do licor em ambos os cenários.

Tabela 3 - Resultado da simulação, após perturbação de $+5 \%$ na vazão de vapor vivo (em negrito: variáveis especificadas (Tabela 1$) ;(\mathrm{e})=$ entrada e $(\mathrm{s})=$ saída)

\begin{tabular}{|c|c|c|c|c|c|c|}
\hline Variável & I & II & III & IV & V & VI \\
\hline \hline Licor & & & & & & \\
\hline \hline Concentração(e) $(\mathrm{kg} / \mathrm{kg})$ & 0,3541 & 0,2330 & 0,1781 & 0,1498 & $\mathbf{0 , 1}$ & 0,1187 \\
\hline Concentração(s) $(\mathrm{kg} / \mathrm{kg})$ & 0,6289 & 0,3541 & 0,2330 & 0,1781 & 0,1187 & 0,1498 \\
\hline Vazão(e) $(\mathrm{kg} / \mathrm{s})$ & 6,7707 & 10,2882 & 13,4623 & 16,0022 & $\mathbf{2 3 , 9 8}$ & 20,1853 \\
\hline Vazão(s) $(\mathrm{kg} / \mathrm{s})$ & 2,8680 & 6,7707 & 10,2882 & 13,4623 & 20,1853 & 16,0022 \\
\hline Temperatura(e) $\left({ }^{\circ} \mathrm{C}\right)$ & 90,1983 & 71,8326 & 58,5037 & 35,6423 & $\mathbf{8 0}$ & 48,4722 \\
\hline Temperatura(s) $\left({ }^{\circ} \mathrm{C}\right)$ & 124,0833 & 90,1983 & 71,8326 & 58,5037 & 48,4722 & 35,6423 \\
\hline EPE $\left({ }^{\circ} \mathrm{C}\right)$ & 20,9217 & 6,9675 & 3,3000 & 2,0746 & 1,1219 & 1,4506 \\
\hline Calor específico $(\mathrm{kg} / \mathrm{kg} \cdot \mathrm{K})$ & 3,5097 & 3,7125 & 3,8150 & 3,8647 & 3,9913 & 3,9389 \\
\hline \hline Vapor & & & & & & \\
\hline \hline Temperatura(e) $\left({ }^{\circ} \mathrm{C}\right)$ & $\mathbf{1 3 9}$ & 103,1615 & 83,2308 & 68,5326 & 56,4290 & 47,3502 \\
\hline Temperatura(s) $\left({ }^{\circ} \mathrm{C}\right)$ & 103,1615 & 83,2308 & 68,5326 & 56,4290 & 47,3502 & 34,1916 \\
\hline Vazão(e) $(\mathrm{kg} / \mathrm{s})$ & $\mathbf{4 , 4 5 8 6}$ & 3,9026 & 3,5174 & 3,1740 & 2,5399 & 3,7946 \\
\hline Vazão(s) $(\mathrm{kg} / \mathrm{s})$ & 3,9026 & 3,5174 & 3,1740 & 2,5399 & 3,7946 & 4,1830 \\
\hline \hline Indice de desempenho & & & & & & \\
\hline \hline Área $\left(\mathrm{m}^{2}\right)$ & $\mathbf{5 5 4 , 4 0 0 0}$ & - & - & - & - & - \\
\hline Consumo de vapor $(\mathrm{kg} / \mathrm{s})$ & 4,4586 & - & - & - & - & - \\
\hline Capacidade de evap. $(\mathrm{kg} / \mathrm{s})$ & 21,1115 & - & - & - & - & - \\
\hline Economia de vapor $(\mathrm{kg} / \mathrm{kg})$ & 4,7349 & - & - & - & - & - \\
\hline
\end{tabular}

\subsection{Análise de Sensibilidade}

O objetivo de um estudo de sensibilidade é avaliar o efeito da incerteza, comum às etapas de modelagem matemática e de estimativa de propriedades e coeficientes, sobre o projeto (dimensionamento) ou a operação (comportamento) real. A Figura 2(b) mostra a Função Sensibilidade da concentração final do licor (C) em relação a vazão de vapor vivo (F), conforme procedimento em Perlingeiro (2005). Mostra-se também o valor da concentração final do licor. Observa-se uma sensibilidade significativa, ora positiva ora negativa (pico), da concentração, principalmente para variações de vapor entre 0 e 5\%. Tal análise é útil para verificar a margem de operação do processo sem perdas significativas de seu desempenho. 


\section{Congresso Brasileiro de Engenharia Química \\ em Iniciação Científica \\ UFSCar - São Carlos - SP

Figura 2 - Resultados: (a) simulação, com perturbações de $\pm 5 \%$ na vazão de vapor vivo, e (b) análise de sensibilidade da concentração final do licor em relação a vazão de vapor vivo.

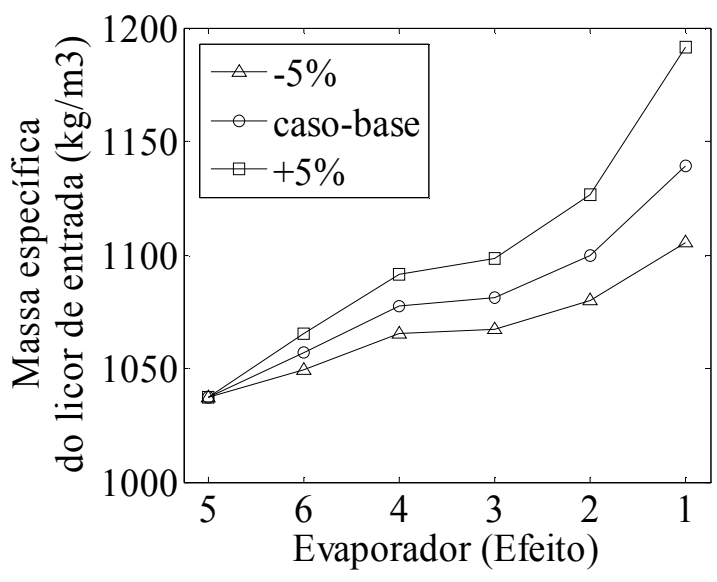

(a) Efeito 5 (entrada) e efeito 1 (saída).

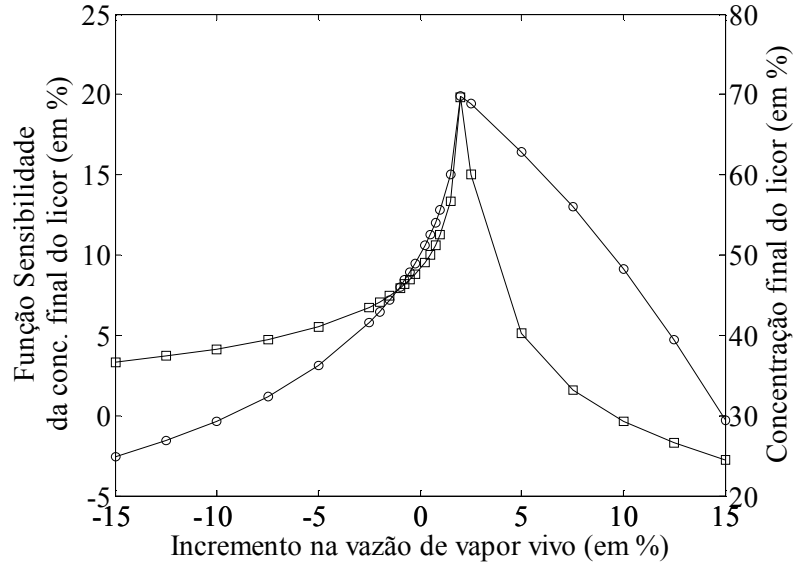

(b) Sensibilidade ( $\square)$ e concentração (०).

\section{CONSIDERAÇÕES FINAIS}

Exploraram-se as etapas de dimensionamento de equipamentos, de simulação de novos cenários, e de análise de sensibilidade de variáveis-chave, após a modelagem matemática e validação de um sistema de evaporação múltiplo efeito de indústrias de celulose Kraft. Ressalta-se o caráter interdisciplinar da área de Engenharia de Processos e sua crescente relevância para a própria manutenção e então expansão do negócio das indústrias químicas.

\section{REFERÊNCIAS}

HOLMLUND, K.; PARVIAINEN, K. Evaporation of black liquor, In: GULLICHSEN, J.; FOGELHOLM, C.J. (eds,) Chemical pulping (book 6B), Cap. 12, Helsinque: Fapet Oy, 1999.

KUMAR, D.; KUMAR V.; SINGH, V. P. Modeling and dynamic simulation of mixed feed multi-effect evaporators in paper industry. Applied Mathematical Modelling, v. 37, p. 384397, 2013.

MASSE, M. A.; KIRAN, E.; FRICKE, A. L. Freezing and glass transition phenomena in polymer-diluent mixtures, Polymer, v. 27, p. 619-622, 1986.

MCCABE, W. L.; SMITH, J. C.; HARRIOT, P. Unit operations of chemical engineering, 7th ed., Singapura: McGrawHill, 2005.

PERLINGEIRO, C. A. G. Engenharia de processos: Análise, simulação, otimização e síntese de processos químicos, 1a ed., São Paulo: Edgard Blücher, 2005.

SENAI (Serviço Nacional de Aprendizagem Industrial); IPT (Instituto de Pesquisas Tecnológicas do Estado de São Paulo S.A.). Celulose e papel: Tecnologia de fabricação da pasta celulósica, vol. 1, 2a ed., São Paulo: IPT, 1988.

VENKATESH, V.; NGUYEN, X. N. Evaporation and concentration of black liquor, In: HOUGH, G. Chemical recovery in the alkaline pulping process, Cap. 3, Atlanta: Tappi, 1985. 